
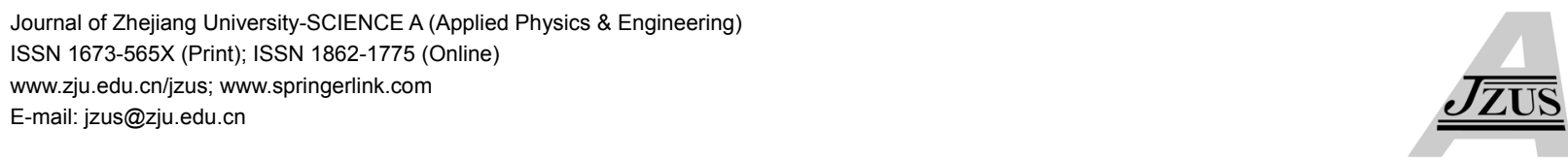

Book review:

\title{
Solar chimney power generating technology
}

Renaud Kiesgen de RICHTER

(National Engineering School of Chemistry in Montpellier, University of Montpellier, Montpellier 34090, France)

E-mail: renaud.derichter@gmail.com

http://dx.doi.org/10.1631/jzus.A17BR001

Ting-zhen MING, 2016. Solar Chimney Power Plant Generating Technology. Academic Press of Elsevier. ISBN 978-012-805370-6

Ting-zhen MING, 2016. Solar Chimney Power Generating Technology. Zhejiang University Press. ISBN 978-7-308$15907-4$

Since Prof. Jorrg SCHLAICH from the University of Stuttgart in Germany built the first solar chimney power plant (SCPP) prototype in the early 1980s, research on SCPP technology has aroused worldwide attention from experiment, to theory and then feasibility studies for large-scale commercial application. However, except for the fewer than 60 -pages piece on the prototype of a SCPP in Manzaneres, Spain, published in 1995 by Prof. Jǒrg SCHLAICH, a complete introduction to the basic principles of SCPP, covering the key technology and cutting-edge progress has been lacking. The publication of the book Solar Chimney Power Generating Technology by Prof. Ting-zhen MING seems to perfectly fill the gap.

Prof. MING's book has nine chapters with 229 pages in total. It is divided into four parts: (1) a brief review of SCPP technology including the principles, experiments, theoretical research, and the future development; (2) the basic operational theory of SCPP technology mainly including the thermodynamic principle of the system and the mechanism of the driving force; (3) the flow and heat transfer performance, turbine design and coupled simulation method the energy storage characteristics of the SCPP system with the energy storage layer, and the interaction mechanism of SCPP and the ambient air flow, ac-

(1) ORCID: Renaud Kiesgen de RICHTER, http://orcid.org/0000-00025669-2267

(c) Zhejiang University and Springer-Verlag Berlin Heidelberg 2017 companied by a miniature indoor SCPP prototype test; (4) a look at the theoretical research frontier of solar chimney power generating systems.

For those readers who have a background in solar energy, heat transfer, and thermodynamics, this book is very easy to read and understand. The main features and innovations of the book lie in three aspects:

1. The thermodynamics cycle theory, the helioaero-gravity effect, structure optimization, the energy storage mechanism, turbine operating and coupling characteristics, and the coupling mechanism between the SCPP and the ambient air flow are analyzed. It covers all the issues of concern to readers, and therefore provides a solid theoretical basis and application guidance for the design and commercial application of large scale SCPP technology.

2. It provides the readers with theoretical SCPP technology research and analysis methods. These methods include the conjugate mathematical model including the fluid flow and heat transfer theories in the collector, chimney, and energy storage layer, the coupling simulation method between the non-rotating collector and the chimney-rotating turbine zone, the porous medium model analysis method of the soil thermal storage layer, and the coupling analysis methods between the solar chimney power generating system and the environment of the air flow inside and outside the system. These analytical methods can provide a good reference for the theoretical analysis of integrated application systems in other fields.

3. Although the last chapter of this book is very short, it concisely provides the readers with the latest cutting-edge theoretical research directions and topics of SCPP technology. If we can completely solve these and other future research problems, this field will form a complete theoretical system.

Note that there are very few studies on real experiments on prototypes in this book. If in the future the author can provide more experimental results of the latest experimental power station-solar chimney power generating system in Inner Mongolia, China, it will be very useful for the scientific community. 